ORIGINAL ARTICLE

\title{
Factors affecting metastases to non-sentinel lymph nodes in breast cancer
}

\section{F J Fleming, D Kavanagh, T B Crotty, C M Quinn, E W McDermott, N O'Higgins, A D K Hill}

See end of article for authors' affiliations

.....................

Correspondence to: Mr A D K Hill, St Vincent's University Hospital, Elm Park, Dublin 4, Ireland; adkhill@ucd.ie

Accepted for publication 3 September 2003

\begin{abstract}
Aims: Because sentinel lymph node (SLN) biopsy for breast cancer has become well established, one of the challenges now is to determine which patients require a completion axillary dissection following a positive SLN biopsy.

Methods: A prospective database of patients who underwent SLN biopsy for invasive breast cancer from July 1999 to November 2002 ( $n=180$ ) was analysed. Fifty four patients $(30 \%$ ) had one or more positive $\mathrm{SLN}$, and all underwent a completion axillary dissection. This subgroup was further analysed to delineate which factors predicted non-SLN metastasis.

Results: Twenty six of the 54 patients with a positive SLN had additional metastases in non-SLNs. Significant variables that predicted non-SLN metastasis included extranodal extension (odds ratio (OR), 17.399; 95\% confidence interval (CI), 1.69 to 178.96) and macrometastasis within the SLN (OR, 6.985; $95 \% \mathrm{Cl}, 1.291$ to 37.785$)$.

Conclusions: In patients with invasive breast cancer and a positive SLN, extranodal extension or macrometastasis within the SLN were both independent predictors of non-SLN involvement.
\end{abstract}

S entinel lymph node (SLN) biopsy has come into widespread use as an accurate staging procedure for early breast cancer. Several studies have documented that SLN biopsy in experienced hands can accurately determine the status of the axillary nodes. ${ }^{1}$ When results of the histopathological examination of SLNs are negative, the remaining non-SLNs in the axilla are unlikely to contain tumour cells. Thus, SLN biopsy offers the advantage of accurately staging the axilla and eliminating the need for a full axillary dissection for patients who have a negative SLN. The optimal treatment for patients with positive SLNs is less clear. In approximately $50-65 \%$ of patients the SLN is the sole site of regional nodal metastasis. ${ }^{2}{ }^{3}$ The aim of our study was to determine factors that predict the presence of further axillary lymph node metastases in patients with a positive SLN.

"Sentinel lymph node (SLN) biopsy offers the advantage of accurately staging the axilla and eliminating the need for a full axillary dissection for patients who have a negative SLN"

\section{METHODS}

A prospective database of patients who underwent SLN biopsy for invasive breast cancer from July 1999 to December 2002 ( $\mathrm{n}=180)$ was analysed. Patients with clinically positive axillae were excluded from our analysis. Our technique for SLN biopsy has been described previously. ${ }^{4}$ Patients received a radiocolloid isotope (2 ml Nanocis) injected intraparenchymally around the tumour or intradermally over the tumour site. Lymphoscintigraphy was performed 90 minutes after injection using a $\gamma$ camera. Scintigraphic images were recorded in the anterior and oblique projections, and the position of the sentinel node was marked on the skin.

Perioperatively, before making the first skin incision, background $\gamma$ counts were taken, as were counts of the breast injection site and the "hottest" point in the axilla. Isosulfan blue dye $(4 \mathrm{ml})$ was injected around the tumour or immediately adjacent to the biopsy cavity if an open biopsy had been performed previously for diagnostic purposes. The SLN was identified using a combination of lymphoscintigraphy, blue dye, and an intraoperative hand held $\gamma$ probe (Neoprobe 2000). Counts were noted of the node(s) in situ and ex vivo, and of the axillary background. A successful radioisotope localisation occurred when the axillary background had counts of $25 \%$ or less compared with the SLN counts ex vivo.

Grossly, the number of SLNs was noted and the order of their dissection was recorded. Each SLN was entirely submitted and routinely processed after 24 hours of formalin fixation. Small SLN nodes $(<5 \mathrm{~mm}$ ) were submitted intact, whereas lymph nodes larger than this were divided into two or more pieces, all embedded and examined with haematoxylin and eosin (H\&E) at three levels, separated by $150 \mu \mathrm{m}$. Unstained sections were prepared at each of these levels and if the H\&E stained slides were negative for metastatic disease, then immunohistochemical staining for cytokeratin (CAM 5.2 antibody) was performed on one of the levels. The sections were immunostained with a mouse monoclonal anticytokeratin CAM 5.2 antibody (Becton Dickinson, Oxford, UK) using a standard peroxidase-antiperoxidase procedure in an automated immunostainer (Ventana Benchbark, Strasburg, France). Appropriate positive and negative controls were incorporated in each run of the immunostainer. The subsequently dissected axillary lymph nodes were stained with H\&E only and examined at one level. A micrometastasis was defined as a tumour deposit smaller than $2 \mathrm{~mm}$ and macrometastasis was defined as a deposit greater than $2 \mathrm{~mm}$. Extranodal extension was considered to be present when tumour was seen outside the lymph node capsule. Extranodal extension was further subdivided into invasion into the hilar fat and invasion extending into the perinodal fat beyond the hilum.

Abbreviations: ALND, axillary lymph node dissection; $\mathrm{H} \& \mathrm{E}$, haematoxylin and eosin; LVI, lymphovascular invasion; SLN, sentinel lymph node 
Fifty four patients had one or more positive SLN, all of whom went on to have a level I, II, and III lymph node axillary dissection (ALND) and form the basis of our study. Multiple variables were analysed in each of the three categories of patient characteristics, tumour characteristics, and SLN characteristics.

The patient characteristics examined were age, tumour location, tumour palpability, and type of operation performed. The primary tumour was analysed with respect to size of the invasive component, histological type, histological grade, oestrogen receptor status, and presence of lymphovascular invasion (LVI). The SLN was examined for size of the metastasis within the node, extranodal extension, and the number of positive SLNs retrieved.

Statistical comparisons were performed with $\chi^{2}$ analysis for categorical variables and multivariate analysis to determine whether any of these characteristics alone or in combination could accurately predict the remaining non-SLN status.

A logistic regression model was used in the multivariate analysis incorporating a backward stepwise regression model. Significance was determined at $\mathrm{p}<0.05$.

\section{RESULTS}

Table 1 outlines the patient and primary tumour characteristics. The median patient age was 53 years, and the median tumour size was $26 \mathrm{~mm}$. Of the 54 patients who underwent completion ALND, 26 had metastases in the non-SLNs, with a median of four (range, 1-26) positive non-SLNs. Table 2 outlines the extent of axillary nodal involvement. Non-SLN metastases were found in $15(53 \%)$ patients with more than one positive SLN $(n=15)$ and in $39(46 \%)$ patients with a single positive SLN. Although increasing tumour size was significantly associated with a positive SLN $(p<0.0001)$, tumour size was not associated with non-SLN metastasis (table 3). Seven of the 54 patients who underwent completion ALND were SLN positive by cytokeratin staining only, and one of these patients had non-SLN metastases.

On univariate analysis, clinicopathological features of the SLN associated with non-SLN metastases included extranodal extension around the SLN and macrometastasis within the SLN. Ten of the 11 patients with extranodal extension (fig 1) around the SLN had non-SLN metastases. Eight of the 11 patients with extranodal extension had invasion into the perinodal fat, whereas the remaining three patients had invasion into the hilar fat only. Patients with extranodal extension into the perinodal fat had a mean of 3.6 positive non-SLNs, whereas the subgroup with extension into the hilar fat had a mean of only 1.6 positive non-SLNs in the completion ALND.

Twenty three of the 38 patients with macrometastasis in the SLN had further disease in the axilla upon completion

Table 1 Patient and primary tumour characteristics

\begin{tabular}{lc}
\hline Characteristic & Number \\
\hline Median age in years (range) & $53(32-83)$ \\
Median tumour size in mm (range) & $26(11-80)$ \\
T1 & $16(30 \%)$ \\
T2 & $33(61 \%)$ \\
T3 & $5(9 \%)$ \\
Median number SLNs harvested (range) & $2(1-4)$ \\
Median number non-SLNs harvested (range) & $23(12-48)$ \\
Invasive ductal carcinoma & $40(74 \%)$ \\
Invasive lobular carcinoma & $8(15 \%)$ \\
Mixed carcinoma & $6(11 \%)$ \\
Grade 1 & $6(11 \%)$ \\
Grade 2 & $30(56 \%)$ \\
Grade 3 & $18(33 \%)$ \\
\hline SLN, sentinel lymph node. & \\
\hline
\end{tabular}

\begin{tabular}{ll}
$\begin{array}{l}\text { Table } 2 \\
(\mathrm{n}=54)\end{array}$ & Distribution of positive axillary lymph nodes \\
\hline Number of positive nodes & Number $(\%)$ of patients \\
\hline 0 & $28(52 \%)$ \\
$1-3$ & $12(22 \%)$ \\
$4-9$ & $8(15 \%)$ \\
$>9$ & $6(11 \%)$ \\
\hline
\end{tabular}

ALND, compared with three of the 16 patients with micrometastasis in the SLN. On multivariate analysis, extranodal extension around the SLN $(p=0.0163)$ and macrometastasis in the SLN $(p=0.024)$ were both independently associated with non-SLN metastases.

Patients with Tl tumours were then analysed separately. On univariate analysis extranodal extension $(\mathrm{p}=0.0293)$ and macrometastasis ( $\mathrm{p}=0.036)$ were significantly associated with non-SLN metastases. On multivariate analysis, neither variable was significant, although macrometastasis was approaching significance $(\mathrm{p}=0.054)$. The three factors associated with SLN only metastasis were Tl tumour size, absence of LVI, and micrometastasis in the SLN. Although none of these factors was sufficient to predict non-SLN metastases alone, none of the six patients who had all three characteristics had disease in the non-SLNs.

\section{DISCUSSION}

SLN biopsy in breast cancer is emerging as a highly sensitive technique for identifying axillary metastasis in a minimally invasive manner. Because the SLNs are the nodes most likely to harbour metastases, a more detailed examination of these nodes can be performed, including extra sections and staining for cytokeratin. This focused approach to the SLN means that if the SLN is found to be negative by both H\&E and cytokeratin staining, then the chance of non-SLN metastases is very low. ${ }^{56}$ Approximately half of all patients with positive SLNs have no residual disease in their axilla, and such patients would not be expected to benefit from a completion ALND. The aim of our study was to identify factors that can predict non-SLN metastases in patients with a positive SLN.

On multivariate analysis, extranodal extension around the SLN was significantly associated with residual disease in the axilla following positive SLN biopsy. Extranodal extension around the SLN was identified in $20 \%$ of patients with SLN metastasis, correlating strongly with non-SLN metastases $(p=0.0163)$, as has been reported previously. ${ }^{7}$ Extranodal extension is usually found at the vascular root of the lymph node hilum involving the efferent lymphatic vessels. ${ }^{8} 9$ Thus, it probably represents tumour cells in transit to other sites. However, although it was significantly associated with nonSLN metastases, it identified only 10 of 26 patients with nonSLN metastases, because it is a relatively uncommon histopathological finding.

The size of tumour deposit in the SLN was also a significant indicator of non-SLN metastases in the regional basin. Twenty three of the 38 patients with SLN macrometastases had non-SLN involvement, compared with three of 16 patients with SLN micrometastases, similar to figures reported previously. ${ }^{2}$ Seven of the total 54 patients had a positive SLN by cytokeratin staining only, one of whom had a non-SLN positive by H\&E staining on ALND. The clinical relevance of positive micrometastases by cytokeratin staining is unknown. Some studies have found no prognostic significance in patients with axillary node micrometastases, whereas others have reported a higher recurrence rate and 


\begin{tabular}{|c|c|c|c|c|}
\hline Feature & No. SLN+ & No. (\%) non-SLN+ & $\begin{array}{l}\text { Univariate analysis } \\
\text { (p value) }\end{array}$ & $\begin{array}{l}\text { Multivariate analysis } \\
\text { (p value) }\end{array}$ \\
\hline $\mathrm{Tlb}$ & 4 & $1(25 \%)$ & 0.5972 & \\
\hline Tlc & 12 & $5(42 \%)$ & 0.610 & \\
\hline $\mathrm{T} 2$ & 33 & $18(55 \%)$ & 0.2382 & \\
\hline T3 & 5 & $2(40 \%)$ & 0.1860 & \\
\hline \multicolumn{5}{|l|}{ Metastasis size } \\
\hline Micrometastasis & 16 & $3(19 \%)$ & & \\
\hline \multirow{2}{*}{\multicolumn{5}{|c|}{ Lymphovascular invasion }} \\
\hline & & & & \\
\hline Present & 37 & $18(49 \%)$ & 0.913 & \\
\hline Absent & 17 & $8(47 \%)$ & & \\
\hline \multicolumn{5}{|l|}{ Extranodal extension } \\
\hline Present & 11 & 10 (91\%) & 0.0015 & 0.0163 \\
\hline Absent & 43 & $16(37 \%)$ & & \\
\hline \multicolumn{5}{|l|}{ Patient age (years) } \\
\hline$<50$ & 18 & $7(39 \%)$ & 0.3356 & \\
\hline$>50$ & 36 & $19(53 \%)$ & & \\
\hline \multicolumn{5}{|c|}{ Primary tumour location } \\
\hline Upper outer quadrant & 18 & $10(55 \%)$ & 0.5920 & \\
\hline Upper inner quadrant & 9 & $4(44 \%)$ & & \\
\hline Lover inner quadrant & 4 & $3(75 \%)$ & & \\
\hline Lower outer quadrant & 7 & $2(29 \%)$ & & \\
\hline Central & 16 & $7(44 \%)$ & & \\
\hline \multicolumn{5}{|l|}{ Histological type } \\
\hline Invasive ductal & 39 & $17(44 \%)$ & 0.1342 & \\
\hline Invasive lobular & 9 & $7(77 \%)$ & & \\
\hline Mixed & 6 & $2(33 \%)$ & & \\
\hline \multicolumn{5}{|l|}{ Grade } \\
\hline 1 & 6 & $2(33 \%)$ & 0.4411 & \\
\hline$\|$ & 30 & $15(50 \%)$ & 0.7607 & \\
\hline III & 18 & $9(50 \%)$ & 0.8473 & \\
\hline
\end{tabular}

decreased survival in such patients. ${ }^{10-12}$ The use of staining for cytokeratin led to the upstaging of $4 \%$ of our SLN population that had been SLN negative by H\&E examination alone.

\section{"On multivariate analysis, extranodal extension around the sentinel lymph node (SLN) was significantly associated with residual disease in the axilla following positive SLN biopsy"}

The precise link between tumour size, LVI, and prediction of non-SLN metastases is an evolving issue with conflicting reports in the literature. ${ }^{13-15}$ Although tumour size and LVI were significantly associated with SLN positivity, they were

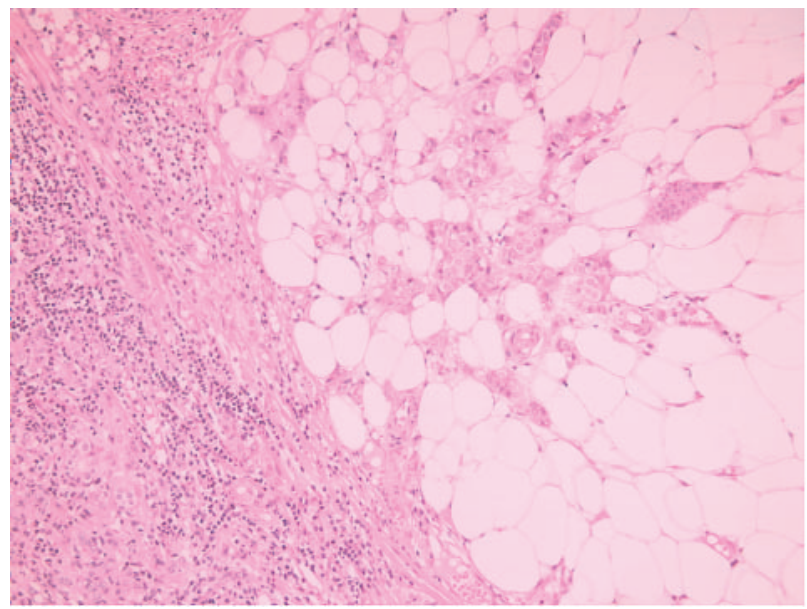

Figure 1 Tumour extending beyond the capsule of a sentinel lymph node using haematoxylin and eosin staining. not predictors of additional positive nodes in our study. It is possible that whereas factors in the primary tumour, such as tumour size and presence of lymphovascular invasion, may be significant in predicting SLN positivity, characteristics of the SLN itself, such as tumour volume and presence of extranodal invasion, may then come into play in predicting further axillary node involvement.

Although all six of the patients with a Tl tumour, SLN micrometastasis, and absence of LVI had SLN metastasis only, no single variable predicted non-SLN metastasis with sufficient sensitivity that ALND could safely be omitted. There was a significant rate of non-SLN metastases among patients with these more favourable tumour characteristics.

One of the four patients with Tlb tumours and three of the 16 patients with SLN micrometastases had non-SLN metastasis.

Several studies have attempted to identify patient subgroups with disease confined to the SLN who thus could have been spared ALND. Reynolds et al observed disease confined to the SLN in 18 patients with $\mathrm{Tl}$ tumours and SLN micrometastasis. They concluded that ALND may not be necessary in patients with $\mathrm{Tl}$ breast tumours and SLN micrometastasis. ${ }^{16}$ Similarly, Chu et al reported that less than $6 \%$ of patients with Tl tumours and SLN micrometastasis had non-SLN involvement, leading the authors to postulate that such patients could possibly be spared a completion ALND. ${ }^{17}$ However, the extent of non-SLN metastases could have been underestimated because the axillary dissection specimen had H\&E examination only. This issue was addressed by the same group, who found that the incidence of non-SLN metastases in patients with Tl tumours and SLN micrometastasis rose from $14 \%$ to $24 \%$ when two level immunohistochemistry was used in addition to $\mathrm{H} \& \mathrm{E}$ examination of the non-SLNs. A subgroup analysis of the patients with T1-T2 tumours, no extranodal extension or lymphovascular invasion, and SLN micrometastasis still 


\section{Take home messages}

- In patients with invasive breast cancer and a positive sentinel lymph node (SLN), extranodal extension or macrometastasis within the SLN were both independent predictors of non-SLN involvement

- T1 tumours with SLN micrometastasis and no lymphovascular invasion were associated with metastasis confined to the SLN

- However, no clinicopathological feature could reliably predict non-SLN metastases such that completion axillary lymph node dissection (ALND) could be safely avoided in some patients

- Until large multicentre trial data have become available, all patients with a positive SLN should be offered ALND

demonstrated a $12 \%$ incidence of non-SLN metastases, despite the favourable characteristics shared by this cohort. ${ }^{7}$

den Bakker et al also reviewed a series of 32 patients with SLN micrometastases and performed cytokeratin staining on the non-SLNs. Eleven patients (34\%) were found to have non-SLN metastasis by cytokeratin and H\&E staining, compared with six patients (19\%) by H\&E examination alone. ${ }^{18}$ There was no subgroup with regard to tumour size or grade identified that did not have non-SLN metastases. Although cytokeratin staining of the non-SLNs is labour intensive and not feasible in routine clinical practice, these studies demonstrate that the true incidence of non-SLN metastases may be underestimated, and that it may not be prudent to extrapolate from relatively small single institution studies that completion ALND can be avoided in certain groups with positive SLNs.

The conflicting reports in the literature emphasise the importance of the ongoing American College of Surgeons' trials in which patients with negative SLNs (by H\&E) are followed without axillary dissection (Z0010), and those with positive SLNs are randomised to ALND or no further axillary treatment (Z0011). ${ }^{19}$ Hopefully, such trials will determine in which patient subgroups, if any, ALND can be safely omitted, and the precise prognostic importance of cytokeratin positive only SLNs.

We found that macrometastasis within the SLN and extranodal extension around the SLN were both significantly associated with non-SLN metastases on multivariate analysis. Although T1 tumours with SLN micrometastasis and no LVI were associated with metastasis confined to the SLN, no clinicopathological feature could reliably predict non-SLN metastases such that completion ALND could be safely avoided in some patients. We conclude that until large multicentre trial data have become available, all patients with a positive SLN should be offered ALND outside ongoing clinical trials.

\section{Authors' affiliations}

F J Fleming, D Kavanagh, T B Crotty, C M Quinn, E W McDermott, N O'Higgins, A D K Hill, Departments of Surgery and Pathology, St

Vincent's University Hospital and Conway Institute of Biomolecular and Biomedical Research, University College Dublin, Ireland

\section{REFERENCES}

1 Strickland AH, Beechey-Newman N, Steer CB, et al. Sentinel node biopsy: an in depth appraisal. Crit Rev Oncol Hematol 2002;44:45-70.

2 Kamath VJ, Giuliano R, Dauway EL, et al. Characteristics of the sentinel lymph node in breast cancer predict further involvement of higher-echelon nodes in the axilla. Arch Surg 2001;136:688-92.

3 Rahusen FD, Torrenga H, van Diest PJ, et al. Predictive factors for metastatic involvement of nonsentinel nodes in patients with breast cancer. Arch Surg 2001; 136:1059-63.

4 Manecksha R, Hill ADK, Dijkstra, et al. Value of sentinel node biopsy in the management of breast cancer. Ir J Med Sci 2001;170:233-8.

5 Turner RR, Ollila DW, Krasne DL, et al. Histological validation of the sentinel lymph node hypothesis for breast carcinoma. Ann Surg 1997:226:271-8

6 Veronesi U, Paganelli G, Viale G, et al. Sentinel lymph node and axillary dissection in breast cancer: results in a large series. J Natl Cancer Inst 1999:91:368-73.

7 Turner RR, Chu KU, Qi K, et al. Pathological features associated with nonsentinel lymph node metastases in patients with metastatic breast carcinoma in a sentinel lymph node. Cancer 2000;89:574-81.

8 Hartveit $F$, Skjaerven R, Maehle BO. Prognosis in breast cancer patients with tumour cells in the efferent vessels of their axillary nodes. J Pathol 1983; 139:379-82.

9 Goldstein NS, Mani A, Vicini F, et al. Prognostic features in patients with stage T1 breast carcinoma and a $0.5-\mathrm{cm}$ or less lymph node metastasis. Am J Clin Pathol 1999;111:21-8.

10 Ludwig Breast Cancer Study Group. Prognostic importance of occult axillary lymph node micrometastases from breast cancers. Lancet 1990;335:1565-8.

11 Clare SE, Sener SF, Wilkins W, et al. Prognostic significance of occult lymph node metastases in node-negative breast cancer. Ann Surg Oncol 1997;4:447-51.

12 Dowlatshahi K, Fan M, Snider HC, et al. Lymph node micrometastasis from breast carcinoma. Cancer 1997;80:1 188-97.

13 Abdessalam SF, Zervos EE, Prasad M, et al. Predictors of positive axillary lymph nodes after sentinel lymph node biopsy in breast cancer. Am J Surg $2001 ; 182: 316-20$

14 Sachdev U, Murphy K, Derzie A, et al. Predictors of nonsentinel lymph node metastasis in breast cancer patients. Am J Surg 2002;183:213-17.

15 Weiser MR, Mongomery LL, Tan LK, et al. Lymphovascular invasion enhances the prediction of non-sentinel node metastases in breast cancer patients with positive sentinel nodes. Ann Surg Oncol 2001;8:145-9.

16 Reynolds C, Mick R, Donohue JH, et al. Sentinel lymph node biopsy with metastasis: can axillary dissection be avoided in some patients with breast cancer? J Clin Oncol 1999;17:1720-6.

17 Chu KU, Turner RR, Hansen NM, et al. Do all patients with sentinel node metastasis from breast carcinoma need complete axillary node dissection? Ann Surg 1999;229:536-41.

18 den Bakker MA, van Weeszenberg Am de Kanter AY, et al. Non-sentinel node involvement in patients with breast cancer and sentinel node micrometastasis; too early to abandon axillary clearance. J Clin Pathol 2002:55:932-5.

19 Grube BJ, Giuliano AE. Observation of the breast cancer patient with a tumour-positive sentinel node: implications of the ACOSOG ZOO11 trial. Semin Surg Oncol 2001;20:230-7. 